Results Compared to unimmunized, treponemal burden by DF in lesion aspirates at Day 19 was significantly lower in animals that received both Natural and Synthetic adjuvant groups. By qPCR, treponemal burden was significantly lower in the Natural group. At day 19 and 30, respectively, the proportion of lesions ulcerating was significantly lower in the Natural group, compared to Unimmunized. At day 30, the proportion of lesions ulcerating in the Natural group was significantly lower than in the Synthetic group. Mean lesion volume was smaller in immunized groups versus unimmunized on days 10-25 post-challenge. RIT indicated the lowest number of disseminated T. pallidum in rabbit tissues from the Natural group, followed by the Synthetic group, then the unimmunized group.

Conclusion Immunization with the three-antigen cocktail significantly attenuates syphilis infection: enhancing $T$. pallidum clearance, promoting lesion healing, and reducing dissemination. In rabbits, Natural adjuvant was more effective than Synthetic adjuvant in inducing protective immunity.

Disclosure No significant relationships.

\section{S12.3 THERAPEUTIC VACCINATION TO TREAT HPV DISEASE: LESSONS LEARNED FROM HIGH GRADE INTRAEPITHELIAL LESIONS}

Margaret M Madeleine*. Fred Hutchinson Cancer Research Center, USA

10.1136/sextrans-2019-sti.59

Malignancies caused by HPV represent a model clinical setting in which to test principles of immunotherapies, and to discover the consequences of interactions between tumors and their attendant immune milieu. A tumor-specific, non-'self' antigenic target is known, as HPV cancers are driven by constitutive and functionally obligate expression of the E6 and E7 viral oncoproteins, which bind and inactivate p53 and $\mathrm{pRb}$, respectively. HPV disease can also be immunogenic; a growing body of evidence demonstrates that HPV-specific T-cell responses can mediate concomitant histologic regression and clearance of detectable virus in subsets of patients who have cervical HSILs. However, HPV researchers are faced with issues common to the development of effective immune-based therapies for every solid tumor, including determining the mechanisms that shape how a tissue microenvironment renders immune cells dysfunctional, deciphering the immunomodulatory effects of other treatment modalities such as targeted therapies, chemotherapy, and radiation, and identifying contributions to immune functional polarization mediated by tissuespecific microbiota. Insights gained from deconvolution of the cervical lesional microenvironment will be discussed.

\section{S12.4 A MUCOSAL CHLAMYDIA TRACHOMATIS VACCINE STIMULATES PROTECTIVE MEMORY T CELLS}

Michael N Starnbach*. Harvard Medical School, Boston, USA

10.1136/sextrans-2019-sti.60

Many non-mucosal vaccines are poorly protective against mucosal pathogens, presumably because they do not generate mucosa-tropic memory cells. Few mucosal vaccines are in clinical use because live vaccine vectors pose safety risks and killed or molecular antigens (Ags) are weak immunogens when applied to intact mucosa. Adjuvants can potentially overcome this poor immunogenicity, however, conventional mucosal adjuvants possess unfavorable safety profiles. We have developed an adjuvanted vaccine against Chlamydia trachomatis. Genital $C t$ infection induced protective immunity that depended on interferon- $\gamma$ (IFN- $\gamma$ ) producing CD4 T-cells, whereas mucosal exposure to UV-inactivated $C t$ (UV-Ct) generated tolerogenic $\mathrm{C} t$-specific regulatory $\mathrm{T}$-cells, resulting in exacerbated bacterial burden upon $\mathrm{Ct}$ challenge. However, mucosal immunization with UV-Ct complexed with chargeswitching synthetic adjuvant particles (cSAP) did not exert the tolerogenic effect of UV-Ct alone but elicited long-lived protection. This differential effect of UV-Ct-cSAP versus UV-Ct was because the former was presented by immunogenic $\mathrm{CD} 11 \mathrm{~b}^{+} \mathrm{CD} 103^{-}$dendritic cells (DCs), while the latter was acquired by tolerogenic CD $11 b^{-} \mathrm{CD} 103^{+}$DCs. Genital protection was achieved after intrauterine or intranasal, but not subcutaneous vaccination and was inducible in conventional and humanized mice. Regardless of vaccination route, UV-Ct-cSAP induced robust systemic memory cells. However, only mucosal vaccination induced a wave of $C t$-specific effector T-cells that seeded the mucosa during the first week and established resident memory $\mathrm{T}$ cells $\left(\mathrm{T}_{\mathrm{RM}}\right)$. Without $\mathrm{T}_{\mathrm{RM}}$, mice were suboptimally protected, even when circulating memory cells were abundant. For optimal Ct clearance, both early seeding by $\mathrm{T}_{\mathrm{RM}}$ and infection-induced recruitment of a second wave of circulating memory cells were required. Thus, using a novel mucosal vaccine platform, we demonstrate that protection against $C t$ depends on synergistic actions of two memory $\mathrm{T}$ cell subsets with distinct migratory properties.

\section{S13 - CONTROVERSIES IN CLINICAL STI CARE}

\section{Tuesday, July 16, 2019 4:15 PM - 5:45 PM}

\section{S13.1 DOES AZITHROMYCIN HAVE A FUTURE IN THE TREATMENT OF GONORRHOEA AND CHLAMYDIAL INFECTION?}

Jane Hocking*. University of Melbourne, Melbourne School of Population and Global Health, Carlton, Australia

\subsection{6/sextrans-2019-sti.61}

Azithromycin, a second generation macrolide antimicrobial, has been widely used as a first line treatment for chlamydia and non-gonococcal urethritis and as part of dual treatment with ceftriaxone for gonorrhoea. Its unique pharmacokinetic properties including its extensive tissue distribution and long half-life, have enabled it to be administered as single dose treatment making it preferred for many STIs, particularly when there are any concerns about treatment adherence. A single dose regimen of azithromycin gives a larger maximum tissue concentration and has more rapid bacterial clearance than longer courses of the same overall dose suggesting that shorter durations of larger doses $(>1 \mathrm{~g})$ will increase treatment efficacy and reduce the induction of resistance. However, the evidence suggests that the efficacy of azithromycin may vary by site of infection, particularly for chlamydia infection, with observational data finding that it might not be as effective for 
rectal chlamydia infections. The pharmacokinetic properties of azithromycin may also contribute to the development of macrolide resistance in other STIs including gonorrhoea and Mycoplasma genitalium. It has extensive tissue distribution with the majority of the drug confined to the intracellular space. After entering the acidic compartments of cells, it becomes trapped leading to its slow release from the tissues contributing to its long half-life. While this is effective for treating chlamydia, the long half-life results in sub-inhibitory levels in the extracellular space, potentially contributing to the development of macrolide resistance in other organisms. This is particularly an issue when new infections are acquired during the two weeks following azithromycin treatment when sub inhibitory concentrations exist. This presentation will discuss how the pharmacokinetic properties of azithromycin affect its efficacy for treating chlamydia infections and whether it should have an ongoing role as part of a dual treatment regimen with ceftriaxone for gonorrhoea infections.

Disclosure No significant relationships.

\section{S13.2 SHOULD ENTERIC INFECTIONS IN MSM ALWAYS BE TREATED?}

Mark Pakianathan*. St George's University Hospital Foundation Trust, HIV and Sexual Health, London, UK

\subsection{6/sextrans-2019-sti.62}

Sexual transmitted enteric infections (STEIs) have been well recognised in gay, bisexual and other men who have sex with men (GBMSM) for over three decades. This presentation will initially provide an overview of the range of enteric pathogens with propensity for sexual transmission in this population.

There has been a recent increase in the numbers of outbreaks of enteric infections reported affecting this population in particular with shigella species and Hepatitis A. These have occurred across borders reflecting internationally mobile GBMSM networks. Recently, multi-drug antimicrobial resistance (AMR) in strains of Shigella sonnei circulating amongst GBMSM in England and USA have been identified challenging standard treatment approaches. The epidemiology of some of these outbreaks, the emergence of AMR and public health responses will be discussed. Outbreaks of shigella have been linked to HIV status and parallel epidemics of gonorrhoea (also linked with AMR), syphilis and Lymphogranuloma venereumin this population of GBMSM.

Whilst the majority of shigella infections in GBMSM will be self-limiting, contextual and clinical factors may lower the threshold for antimicrobial treatment. The presentation highlights some of these challenges and dilemmas in clinical management particularly in the face of co-infection with STIs, the emergence of AMR and syndemic health inequalities such as problematic chemsex (use of psychoactive substances with sex).

The presentation will conclude with research gaps, implications for policy on STEIs and emphasize the need for partnership working across public health, microbiology and relevant frontline clinical services. The need for management approaches that are holistic and consider wider syndemic health needs when managing patients will also be highlighted.

Disclosure No significant relationships.
S13.3 DO RECTAL BACTERIAL STIS IN WOMEN MATTER? WHO SHOULD WE TEST AND WHEN?

Christine Khosropour*. University of Washington, Epidemiology, Seattle, USA

\subsection{6/sextrans-2019-sti.63}

Rectal bacterial STIs are increasingly recognized as common infections among clinic-attending women, with estimated prevalences of $5 \%$ for rectal Neisseria gonorrhoeae (GC) and 9\% for rectal Chlamydia trachomatis (CT). Although these prevalences are similar to urogenital GC and CT among these same populations of women, we know very little about the health implications or epidemiology of rectal STIs among women. Rectal STIs are typically asymptomatic and the infections themselves may not be morbid conditions. However, some investigators have hypothesized that women could autoinoculate bacteria from the rectum to the vagina which may result in reproductive tract sequelae in the absence of vaginal sex. Even if there were strong evidence to suggest this does occur, it is unclear which populations of women should be targeted for rectal screening. Employing current screening guidelines for urogenital infections would assure treatment of women with concurrent rectal STI, but would miss women with isolated rectal STI. Further, the efficacy of azithromycin for CT may be lower in the rectum than the urogenital tract, suggesting that screening and treating women for urogenital CT without regard to the presence of rectal CT may result in a persistent rectal infection. Alternatively, rectal STI screening could be limited to women who report anal sex. However, the prevalence of rectal STIs is similar among women who do and do not report anal sex, suggesting that this screening strategy would miss a substantial proportion of cases. Finally, some have hypothesized that oral acquisition of CT may lead to rectal infection. If this route is possible, rectal screening for women who report penile-oral sex may be warranted. This session will review the epidemiologic and microbiologic evidence on these topics and will discuss what studies are needed to address the gaps in our understanding of these infections and define a way forward.

Disclosure No significant relationships.

\section{S13.4 HPV VACCINATION IN MSM: WHO SHOULD BE VACCINATED AND IS THERE A ROLE FOR VACCINATION OF OLDER AND/OR HIV-POSITIVE MSM IN PREVENTING INITIAL, PERSISTENT AND RECURRENT HPV AND RELATED DISEASES?}

David Templeton*. RPA Sexual Health, Sydney, Australia

\subsection{6/sextrans-2019-sti.64}

Background Anogenital infection with human papillomavirus (HPV) disproportionately affects men who have sex with men (MSM), especially those living with HIV. It remains unclear whether HPV vaccination of older MSM and/or MSM living with HIV is beneficial in terms of preventing new HPV infections, reinfections with the same HPV subtype, new diagnoses or recurrence of HPV-related lesions or anal cancer.

Results HPV16 causes most anal squamous cell cancer worldwide. However, other high-risk HPV (hrHPV) types contained in the 9-valent vaccine (9vHPVvax) cause a substantial minority of anal cancers in HIV-positive MSM. In the landmark 